\title{
Enhanced drug loading capacity of polypyrrole nanowire network for controlled drug release
}

Shuhui Jiang, Yanan Sun, Xin Cui, Xiang Huang, Yuan He, Shan Ji, Wei Shi and Dongtao Ge

\begin{abstract}
For a conducting polymer (CP) based drug release system, drug loading is often accomplished by a doping process, in which drug is incorporated into polymer as dopant. Therefore, the drug loading capacity is relatively low and the range of drugs can be loaded is limited. In the present work, a polypyrrole (PPy) nanowire network is prepared by an electrochemical method and it is found that the micro- and nanogaps among the individual nanowires of the PPy nanowire network can be used as reservoir to store drugs. Therefore, the drug loading capacity is dependent on the volume of these micro- and nano-vacancies, instead of the doping level. The range of loaded drugs also can be theoretically extended to any drugs, instead of only charged dopants. In fact, it is confirmed here that both hydrophilic and lipophilic drugs can be loaded into the micro- and nano-gaps due to the amphilicity of the PPy nanowire network. As a result, both drug loading capacity and the range of drugs can be loaded are significantly improved. After being covered with a protective PPy film, controlled drug release from the prepared system is achieved by electrical stimulation (cyclic voltammetry, CV) and the amount of drug released can be controlled by changing the scan rate of $\mathrm{CV}$ and the thickness of the protective PPy film.
\end{abstract}

\section{Introduction}

Nanostructured conducting polymers (CPs) have been one of the focus research topics in the field of nanoscience for a number of years. To date, numerous nanostructures of CPs, such as nanowire, nanowire network, nanorod, nanotube, nanoparticle, and nanobelt have been synthesized and studied [1-4]. Among these $\mathrm{CP}$ nanostructures, nanowire networks of CPs, for example, polypyrrole (PPy) nanowire network, have attracted a great deal of interest due to their wide applications in the fields of biosensors, energy source, and electronics [3,4]. However, there are few reports on the use of PPy nanowire work for controlled drug release. Recently, our group extended the application of PPy nanowire network to this field, obtaining an effective drug release system with high adenosine triphosphate (ATP) release efficiency [5]. 
It is well known that CPs can be used as drug carriers due to their unique dopingdedoping and stimulus-responsive property [6-22], where drugs can be incorporated into CPs as dopants and then be released upon electrical stimulation. Therefore, the capacity of drug loading is dependent on the doping level (which is usually low) and the type of drugs is restricted to the charged and small molecules. Actually, relatively low drug loading capacity and limited range of drugs can be loaded have become two main obstacles for the practical applications of the current existing CP-based drug release systems.

In the present work, we find that the micro- and nano-gaps among the individual nanowires of the above mentioned PPy nanowire network can be used as reservoir to store drugs. Because the space for drug storage has changed from the backbones of the CPs to the vacancies among the nanowires, it is not necessary to consider the charge and the volume of the loaded drugs. Therefore, both the drug loading capacity and the range of loaded drugs can be improved significantly. After being covered with a protective PPy film for inhibiting drug leakage, controlled drug release from the prepared $\mathrm{P}-\mathrm{P}$ system (drug-loading PPy nanowire network coated by a PPy film) is achieved by electrical stimulation (cyclic voltammetry, CV) and the amount of drug released can be controlled by changing the scan rate of $\mathrm{CV}$ and the thickness of the protective PPy film.

\section{Experimental \\ 2.1 Chemicals}

Pyrrole, para-toluene sulphonic acid sodium salt (p-TS), iron (III) chloride hexahydrate $\left(\mathrm{FeCl}_{3} \cdot 6 \mathrm{H}_{2} \mathrm{O}\right)$, and dimethyl sulfoxide (DMSO) were obtained from Sinopharm Chemical Reagent Co., Ltd. (China). Pyrrole was distilled under the protection of nitrogen gas and stored at $-20{ }^{\circ} \mathrm{C}$. Hexadecane was got from Aladdin Chemistry Co., Ltd. (China). ATP and dexamethasone (Dex) were purchased from Sangon Biotech Co., Ltd. (China). Dopamine hydrochloride was bought from Sigma. Hydroxycamptothecin (HCPT) and paclitaxel were obtained from Li Shizhen Medicine Group Co., Ltd. (China). All other chemicals were of analytical grade and were used as received. All solutions were prepared using deionized Milli-Q water (Millipore).

\subsection{Preparation of drug loaded PPy nanowire network}

The PPy nanowire network was synthesized electrochemically at room temperature with conventional three-electrode cell by the use of CHI6ooD Electrochemical Workstation (Shanghai $\mathrm{CH}$ instruments, China). A bare gold ( $\mathrm{Au}$, diameter of 4.0 $\mathrm{mm}$ ) was used as working electrode. The counter electrode was a platinum wire and a saturated calomel electrode (SCE) was used as the reference electrode. The electrolyte was an aqueous solution of $0.145 \mathrm{M}$ pyrrole, $0.085 \mathrm{M} \mathrm{p}$-TS and $0.200 \mathrm{M}$ phosphate buffer solution ( $\mathrm{pH}$ 7.40). Before the electropolymerization, the electrolyte solution was degassed with a nitrogen stream for $5 \mathrm{~min}$. PPy nanowire network was grown for $1600 \mathrm{~s}$ from the surface of the Au electrode at $0.477 \mathrm{~mA} \mathrm{~cm}^{-2}$.

\section{http://repository.uwc.ac.za}


After polymerization, the working electrode was removed from the electrolyte and rinsed thoroughly with de-ionized water, and then dried in air at room temperature.

6.0 $\mu \mathrm{L}$ aqueous solution containing 0.01 M ATP (or 0.01 M SSA, 0.02 M Dopamine) or $10 \mu \mathrm{L}$ DMSO solution containing 0.005 M Dex (or 6.0 $\mu \mathrm{L}$ DMSO of 0.01 M HCPT, $5 \mu \mathrm{L}$ DMSO of $0.01 \mathrm{M}$ Pacitaxel) was then dropped onto the surface of the PPy nanowire network and dried in air.

\subsection{Synthesis of the protective PPy film by chemical vapor deposition (CVD) method}

To prevent the stored drugs from leaking, a PPy film was polymerized on the top of the PPy nanowire network by CVD technique. The PPy nanowire network-coated Au electrode was mounted in a reaction vessel equipped with a sealing apparatus and a monomer injection port. 10, 15 and $20 \mu \mathrm{L}$ ethanol solution containing $0.1 \mathrm{M} \mathrm{FeCl}_{3}$ were dropped onto PPy nanowire network-coated Au electrode, respectively. The reaction chamber was vacuumized at room temperature until the internal pressure reduced to $10^{-2}$ Torr. Then, excessive pyrrole monomer was injected into the reactor with a micro-syringe. The pyrrole monomers were polymerized on the surface of the PPy nanowire network at $60^{\circ} \mathrm{C}$ for $1 \mathrm{~h}$. Subsequently, the obtained P-P system was washed with deionized water for several times and dried in air.

\subsection{Characterization and measurements}

The surface morphologies of the PPy nanowire network and the $\mathrm{P}-\mathrm{P}$ system were examined by a scanning electron microscope (SEM, LEO1530, Germany) operated at $20 \mathrm{kV}$. The wettability measurement of the PPy nanowire network was performed on a KRUSS FM 40 MK2 contact angle (CA) goniometer. The static contact angles were obtained using $3 \mu \mathrm{L}$ water droplets or hexadecane droplets. Cyclic voltammetry (CV) test was performed from -0.9 to $0.6 \mathrm{~V}$ sweeping at a constant scan rate of $0.01 \mathrm{~V} / \mathrm{s}$. Fourier transform infrared (FTIR) spectroscopy was recorded on an Avatar 360 spectrophotometer (Thermo Nicolet, USA).

\subsection{Drug release}

Electrical stimulation of ATP release was carried out in $2 \mathrm{~mL} \mathrm{NaCl}$ solution (0.9\%) stirred with a magnetic stirring bar at room temperature, and performed by cycling the potential from -0.9 to $+0.6 \mathrm{~V}$ at different scan rates. Samples were taken at specific times from the release medium, analyzed for ATP content by a UV/Vis Spectrophotometer (UV-1750, Shimadzu, Japan) at $259 \mathrm{~nm}$, and replaced with an equal volume of fresh $0.9 \% \mathrm{NaCl}$ solution. The spontaneous release of ATP was conducted in the same release medium except that the external electrical stimulation was not applied. 
Electrical stimulation of Dex release was carried out in $6 \mathrm{~mL} \mathrm{NaCl}$ solution (0.9\%) stirred with a magnetic stirring bar at room temperature, and performed by cycling the potential from -0.9 to $+0.6 \mathrm{~V}$ at a scanning rate of $100 \mathrm{mV} / \mathrm{s}$. Samples were taken at specific times from the release medium, analyzed for Dex content by the same $\mathrm{UV} / \mathrm{Vis}$ Spectrophotometer at $242 \mathrm{~nm}$, and replaced with an equal volume of fresh $0.9 \% \mathrm{NaCl}$ solution. The spontaneous release of Dex was conducted in the same release medium except that the external electrical stimulation was not applied.

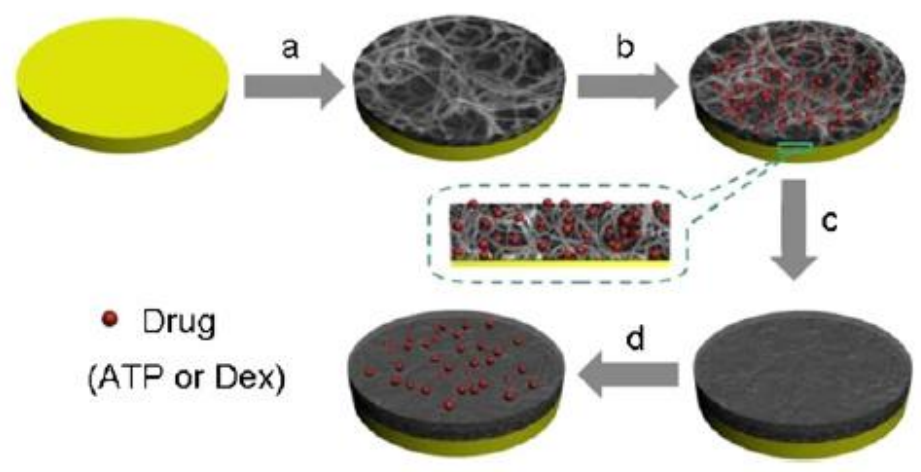

Scheme 1. Schematic process of the fabrication and the drug release of the P-P system. (a) Electropolymerize PPy nanowire network; (b) add drug; (c) prepare PPy film by CVD method; (d) apply electrical stimulation to release drug.

\section{Results and discussion}

Scheme 1 shows the schematic representation of the synthesis and the drug release processes of the P-P system. In the first step (Scheme 1a), PPy nanowire network was synthesized by a simple one-step electrochemical method. SEM images of the PPy nanowire network are shown in Fig. 1. It can be seen clearly that the PPy nanowire network exhibits porous interwoven structures, which inspires us to explore whether drugs can be loaded within the micro- and nano-gaps of the porous nanowire network.

For this purpose, the surface wettability of the PPy nanowire network was examined. As shown in Fig. 2A, when a droplet of water $(3 \mu \mathrm{L})$ was deposited on the surface of the nanowire network, the water will penetrate into the vacancies among individual nanowires and the $\mathrm{CA}$ is about $5^{\circ}$, which indicates a superhydrophilic behavior. In addition, the lipophilic property of the nanowire network was also investigated. It was found that the CA of hexadecane on the surface of nanowire network was about $18^{\circ}$ (Fig. 2B), showing excellent lipophilicity. Therefore, the prepared PPy nanowire network shows amphilicity and it is reasonable to deduce that both hydrophilic and lipophilic drugs can be loaded into the nanowire network. Here, several drugs, including hydrophilic drugs such as ATP, SSA, and Dopamine and lipophilic drugs such as Dex, HCPT and paclitaxel were examined and it was found that all of these drugs, either hydrophilic or lipophilic, could be loaded easily into the nanowire network. In the 
following experiments, ATP and Dex were chosen as the model hydrophilic and lipophilic drugs respectively for various tests (Scheme $1 \mathrm{~b}$ ).

To prevent the stored drug from leaking, a protective PPy film on the surface of the PPy nanowire network should be required.
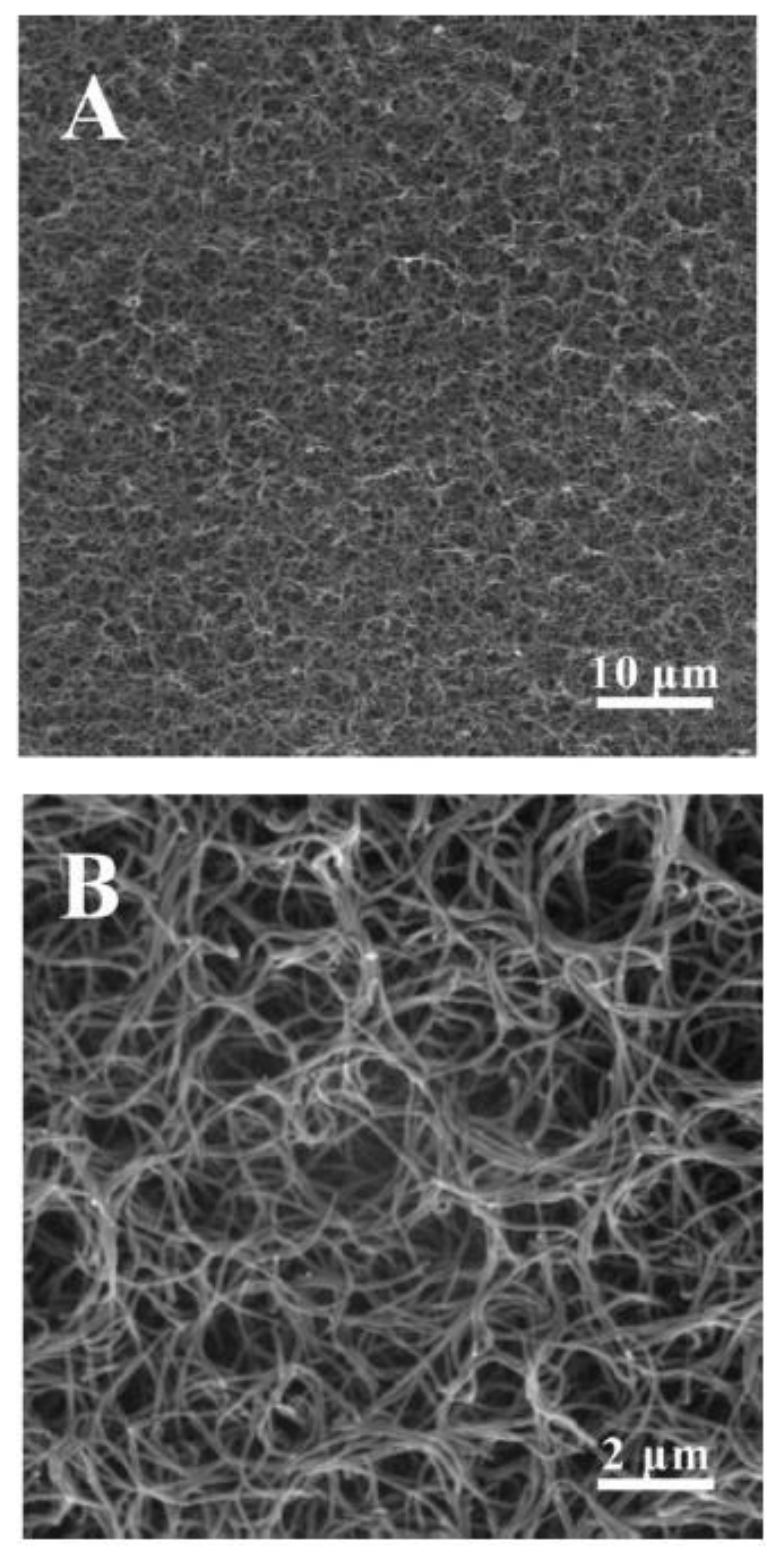

Fig. 1. SEM images of PPy nanowire network: (A) low magnification image; (B) high magnification image.

However, if the film was prepared in aqueous solution, some of the loaded drug would diffuse to the solution due to the concentration difference. Therefore, it is difficult to quantify the loaded drug. To solve this problem, a protective PPy film was polymerized on the surface of the nanowire network by CVD method in our experiment (Scheme 1c). Because the PPy film was formed in the pyrrole vapor under vacuum and no solution was used, drug leakage was prohibited completely. Fig. 3 shows the 
morphology of the protective PPy film. It demonstrates that the film has a dense and smooth surface and the nanostructures were faintly visible.

After covering the nanowire network with the protective film, the whole $\mathrm{P}-\mathrm{P}$ system with ATP as model hydrophilic drug were characterized qualitatively by FTIR spectroscopy in the range of $400-4000 \mathrm{~cm}^{-1}$. The results are presented in Fig. 4 . The absorption bands at $1568,1477,1331$ and $1078 \mathrm{~cm}^{-1}$ are attributed to the PPy chain. The peak around $1568 \mathrm{~cm}^{-1}$ is associated with the pyrrole ring of the combination of C C and C C stretching vibrations. The band at $1477 \mathrm{~cm}^{-1}$ is assigned to the $\mathrm{C} \mathrm{N}$ stretching vibration. The absorption peaks near 1078 and $1331 \mathrm{~cm}^{-1}$ are attributed to the deformation vibrations of $\mathrm{C} \mathrm{H}$ and $\mathrm{C} \mathrm{N}$ stretching vibrations, respectively. The presence of ATP in the PPy nanowire network is proved by the appearance of peaks at $1250 \mathrm{~cm}^{-1}$ (corresponding to the $\mathrm{P} \mathrm{O}$ stretching vibration) and $1085 \mathrm{~cm}^{-1}$ (corresponding to the $\mathrm{P} \mathrm{O}$ stretching vibration). The absorption peaks at 2925 and $2851 \mathrm{~cm}^{-1}$ were intensified due to the introduction of $\mathrm{CH}_{2}$ and $\mathrm{CH}$ groups in ATP.

The electrochemical property of the PPy nanowire network was evaluated by cyclic voltammetry in $0.9 \% \mathrm{NaCl}$ aqueous solution (the naked $\mathrm{Au}$ electrode was also studied for comparison). As shown in Fig. 5, the current of naked Au electrode is close to zero and no obvious redox peaks were observed. In contrast, the PPy nanowire network has clearly redox peaks, which elucidate that PPy nanowire network has much better electrochemical activity than the naked $\mathrm{Au}$ electrode and thus benefits to subsequent drug release.
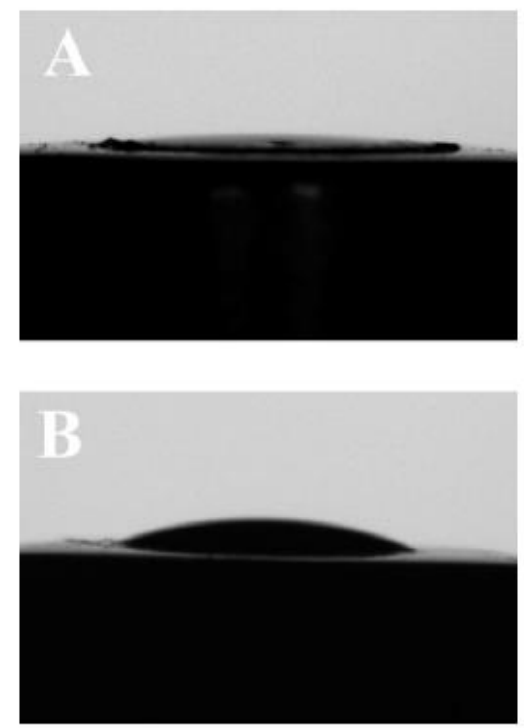

Fig. 2. Water (A) and hexadecane (B) contact angles of PPy nanowire network. 


\section{$10 \underline{\underline{m}}$}

Fig. 3. SEM image of a protective PPy film synthesized by consuming $10 \mu$ L oxidant solution.

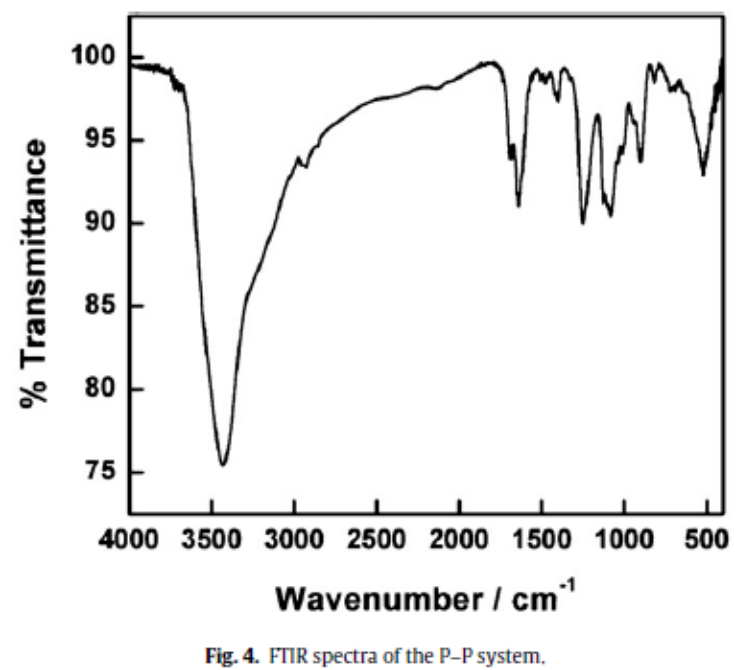

After the deposition of the protective PPy film on the PPy nanowire network, spontaneous release of ATP from this $\mathrm{P}-\mathrm{P}$ system at open circuit was investigated (spontaneous release of ATP from PPy nanowire network was also examined for comparison). ATP release efficiency was plotted versus time, as shown in Fig. 6. It is observed that spontaneous ATP release from nanowire network is rather significant. Nearly 99\% of loaded ATP was released within $1 \mathrm{~h}$. However, only $18 \%$ of ATP was released at open circuit within $10 \mathrm{~h}$ from the $\mathrm{P}-\mathrm{P}$ system, which indicated that the presence of the outer protective PPy film could significantly inhibit the spontaneous ATP release.

Then ATP release from the $\mathrm{P}-\mathrm{P}$ system under CV electrical stimulation (Scheme $1 \mathrm{~d}$ ) was investigated. As shown in Fig. 6, it was found that the effect of scan rate of CV on the ATP release efficiency is significant. About 57, 89 and 95\% of ATP were released from 
the $\mathrm{P}-\mathrm{P}$ system at scanning rates of 50,100 , and $200 \mathrm{mV} / \mathrm{s}$ within $10 \mathrm{~h}$, respectively. The results reveal that like the normal CP-based drug release systems (drugs were loaded by doping), drug release from the $\mathrm{P}-\mathrm{P}$ system also can be performed by electrical stimulation and the drug release rates can be regulated by changing the CV scan rates. It is well known that when PPy was switched from the oxidized state to the reduced state, it was accompanied by a change in the PPy volume, from its expansion state to the contraction state [22,23]. Therefore, the diminishing of the PPy volume could provide the driving force for the expulsion of ATP. The faster the scan rate, the more frequent the contraction and expansion of the film. As a result, more drugs were squeezed out.

On the other hand, since the space for the CVD reaction is fixed, if the amount of the pyrrole is excessive and the polymerization time is long enough, the thickness of the outer protective PPy film will be proportional to the oxidant consumption. In our experiment, three protective PPy films with different thicknesses were prepared by changing the oxidant amounts. As expected, it was observed that the amount of ATP released decreased with the increase of the thickness of the outer protective PPy film. About 91, 66, and 44\% of ATP were released from the systems with the protective PPy films synthesized by consuming 10,15 , and $20 \mu \mathrm{L}$ oxidant solutions $\left(0.1 \mathrm{M} \mathrm{FeCl}_{3}\right)$ in $12 \mathrm{~h}$, respectively, as shown in Fig. 7 .

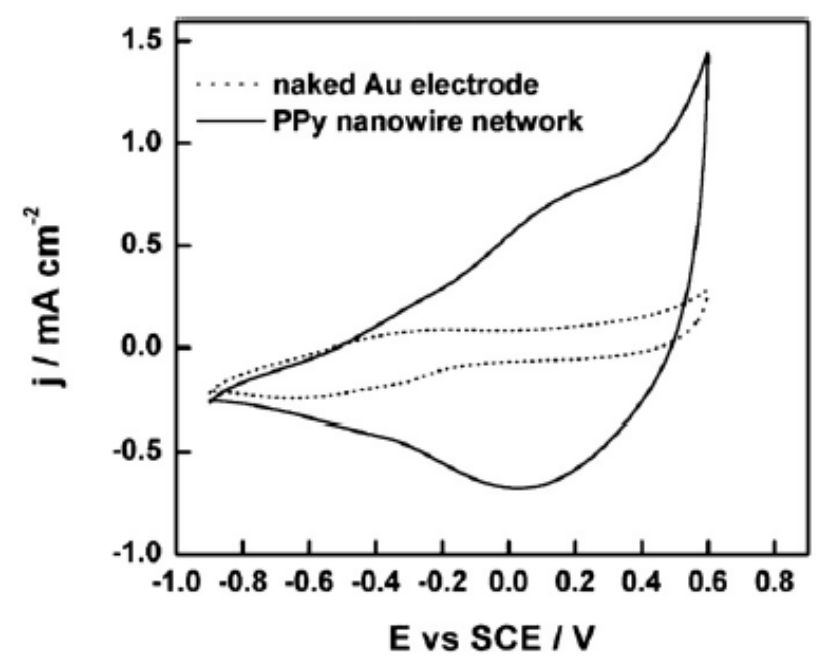

Fig. 5. Cyclic voltammograms of the naked Au electrode and the PPy nanowire network at a scanning rate of $10 \mathrm{mV} / \mathrm{s}$ in $0.9 \% \mathrm{NaCl}$. 


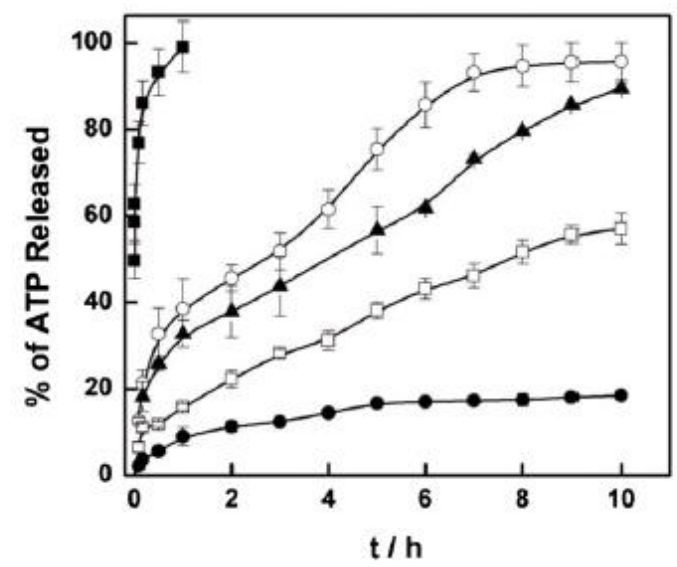

Fig. 6. Percent release of ATP from P-P system (with the protective PPy film synthesized by consuming $10 \mu$ L oxidant solution) upon CV electrical stimulation from -0.9 to $0.6 \mathrm{~V}$ at a scanning rate of $50 \mathrm{mV} / \mathrm{s}(\square), 100 \mathrm{mV} / \mathrm{s}(\mathbf{\Lambda})$ and $200 \mathrm{mV} / \mathrm{s}(0)$, respectively, and from PPy nanowire network ( $\mathbf{\square})$ and P-P system $(\bullet)$ without electrical stimulation,

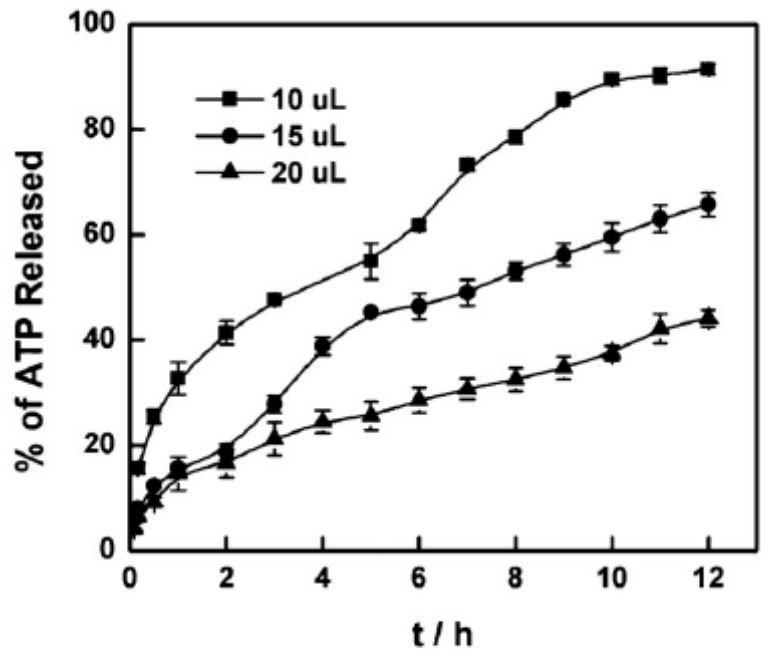

Fig. 7. Percent release of ATP upon CV electrical stimulation from -0.9 to $0.6 \mathrm{~V}$ at a scanning rate of $100 \mathrm{mV} / \mathrm{s}$ from P-P system with the outer protective PPy films

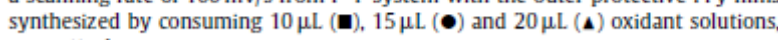
respectively.

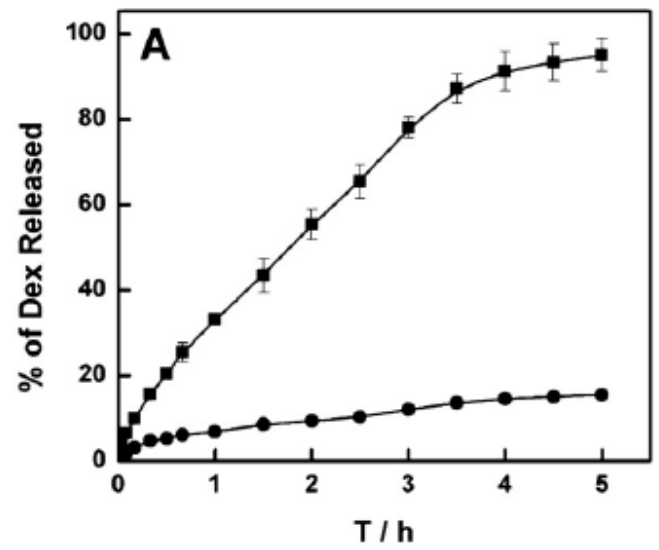

Fig. 8. Percent release of Dex upon electrical stimulation from $-0,9$ to $0.6 \mathrm{~V}$ at a scanning rate of $100 \mathrm{mV} / \mathrm{s}(\mathbf{-})$ and spontaneous release $(\bullet)$ from the P-P systems with the same thickness of the outer protective PPy film that synthesized by consuming $10 \mu \mathrm{L}$. oxidant solution, 
These results revealed that the ATP release efficiency also can be tuned by changing the thickness of the protective PPy film.

In our experiment, release of lipophilic drug Dex from the $\mathrm{P}-\mathrm{P}$ system was also examined. The results present in Fig. 8 show that 95\% of Dex was released under CV electrical stimulation from -0.9 to $0.6 \mathrm{~V}$ at a scanning rate of $100 \mathrm{mV} / \mathrm{s}$ while only $15 \%$ of Dex was diffused from the system at open circuit, indicating that the release of lipophilic drug could also be controlled effectively by the present system.

\section{Conclusion}

A PPy-based drug release system was developed by deposition of a protective PPy film on the surface of PPy nanowire network. The PPy nanowire network was synthesized by a simple one-step electrochemical method. It was found that micro- or nanogaps among the individual nanowires could be used as reservoirs to store drugs. Interestingly, both hydrophilic and lipophilic drugs could be loaded into the system by taking advantage of amphilicity of the PPy nanowire network and subsequently be released upon electrical stimulation. The amount of the drug released could be controlled by changing the scan rate of the $\mathrm{CV}$ and the thickness of the outer protective PPy film. In summary, the drug release system prepared here not only keeps the advantage of the conventional PPy-based drug release system (drug release can be performed by electrical stimulation), but also provides some new merits. For example, higher drug loading capacity is achieved and more type of drugs can be loaded. Furthermore, other related functional materials such as drug-loading nanoparticles may also be stored within the PPy nanowire network and thus offer a new strategy for easily incorporation of drugs or drug carriers. It is expected that this PPy nanowire network based drug release system may have great potential for future clinical applications where high drug loading capacity is required.

\section{Acknowledgments}

This work was supported by the National Nature Science Foundation of China (Nos. 31070845, 31271009, 81271689), the Fundamental Research Funds for the Central Universities (No. 2011121001) and the Natural Science Foundation of Fujian Province (No. 2011Jo1331). 


\section{References}

[1] J. Stejskal, I. Sapurina, M. Trchova, Progress in Polymer Science 35 (2010) 1420.

[2] D. Li, J.X. Huang, R.B. Kaner, Accounts of Chemical Research 42 (2009) 135.

[3] C. Li, H. Bai, G.Q. Shi, Chemical Society Reviews 38 (2009) 2397.

[4] H.D. Tran, D. Li, R.B. Kaner, Advanced Materials 21 (2009) 1487.

[5] X.N. Ru, W. Shi, X. Huang, X. Cui, B. Ren, D.T. Ge, Electrochimica Acta 56 (2011) 9887.

[6] Y. Cho, R.B. Borgens, Langmuir 27 (2011) 6316.

[7] D. Svirskis, J. Travas-Sejdic, A. Rodgers, S. Garg, Journal of Controlled Release 146 (2010) 6.

[8] D.T. Ge, R.C. Qi, J. Mu, X.N. Ru, S.M. Hong, S. Ji, V. Linkov, W. Shi, Electrochemistry Communications 12 (2010) 1087.

[9] Y.H. Li, R.J. Ewen, S.A. Campbell, J.R. Smith, Journal of Materials Chemistry 22 (2012) 2687.

[10] C.Y. Wang, P.G. Whitten, C.O. Too, G.G. Wallace, Sensors and Actuators B: Chemical 129 (2008) 605.

[11] R. Wadhwa, C.F. Lagenaur, X.T. Cui, Journal of Controlled Release 110 (2006) 531.

[12] D.T. Ge, X.D. Tian, R.C. Qi, S.Q. Huang, J. Mu, S.M. Hong, S.F. Ye, X.M. Zhang, D.H. Li, W. Shi, Electrochimica Acta 55 (2009) 271.

[13] S. Geetha, C.R.K. Rao, M. Vijayan, D.C. Trivedi, Analytica Chimica Acta 568 (2006) 119.

[14] D.T. Ge, X.N. Ru, S.M. Hong, S.H. Jiang, J. Tu, J. Wang, A.F. Zhang, S. Ji, V. Linkov, B. Ren, W. Shi, Electrochemistry Communications 12 (2010) 1367.

[15] L. Leprince, A. Dogimont, D. Magnin, S. Demoustier-Champagne, Journal of Materials Science: Materials in Medicine 21 (2010) 925.

[16] L.D. Li, C.B. Huang, Journal of the American Society for Mass Spectrometry 18 (2007) 919.

[17] J.C. Wu, W.M. Mullett, J. Pawliszyn, Analytical Chemistry 74 (2002) 4855.

[18] G. Bidan, C. Lopez, F. Mendes-Viegas, E. Vieil, A. Gadelle, Biosensors and Bioelectronics 10 (1995) 219.

[19] K. Kontturi, P. Pentti, G. Sundholm, Journal of Electroanalytical Chemistry 453 (1998) 231.

[20] M. Pyo, J.R. Reynolds, Chemistry of Materials 8 (1996) 128.

[21] B.C. Thompson, S.E. Moulton, R.T. Richardson, G.G. Wallace, Biomaterials 32 (2011) 3822.

[22] X.L. Luo, X.T. Cui, Electrochemistry Communications 11 (2009) 402.

[23] L.M. Lira, S.I. Cordoba de Torresi, Electrochemistry Communications 7 (2005) 717. 